\title{
Uma retomada da discussão sobre a sustentabilidade da política fiscal do Rio Grande do Sul
}

\author{
Liderau dos Santos Marques Júnior \\ Pesquisador na Fundação de Economia e \\ Estatística Siegfried Emanuel Heuser (FEE) \\ Paulo de Andrade Jacinto \\ Professor da PUCRS e pesquisador CNPq
}

\section{Palauras-chave}

equilíbrio orçamentário intertemporal, testes de cointegração, política fiscal.

\section{Classificação JEL E62, C2,} $\mathrm{H} 72, \mathrm{H} 74$.

\footnotetext{
Keywords

intertemporal budget equilibrium, co-integration tests, fiscal policy.

\section{JEL Classification E62, C2,}

H72, H74.
}

\section{Resumo}

Este artigo examina a sustentabilidade da política fiscal do Estado do Rio Grande do Sul para os períodos de 1970 a 1997 e 1970 a 2003. No período de 1970 a 2003 a política fiscal se caracterizou por sucessivos déficits primários e pela acumulação de dívida pública. Embora tenha passado por períodos de deterioração da situação fiscal, o Estado jamais decretou o não-pagamento da dívida, o que sugere que a política fiscal foi sustentável nesse período. A partir do uso de testes de cointegração verificou-se evidências favoráveis à hipótese da sustentabilidade da política fiscal durante os períodos analisados.

\section{Abstract}

This paper investigates the sustainability of the fiscal policy in the state of Rio Grande do Sul for two periods: 1970 to 1997 and 1970 to 2003. From 1970 to 2003, the fiscal policy was characterized by primary deficits and the accumulation of public debt. Regardless of the deterioration of its tax situation, the State never determined the non-payment of its public debt, which suggests the sustainability of the fiscal policy during the two periods. Co-integration tests support the evidence of sustainability during the periods in question. 


\section{1_Introdução}

A política fiscal de um governo é sustentável se a dívida em dado período de tempo é igual ao valor presente dos superávits primários futuros. Em outras palavras, a política fiscal é sustentável se uma dada sequência de dívida pública satisfaz à condição de equilíbrio orçamentário intertemporal.

Segundo Hamilton e Flavin (1985), Wilcox (1989), Trehan e Walsh (1991), a política fiscal de um governo é sustentável se as séries das variáveis "déficit público" e "dívida pública" são estacionárias. Os autores referidos utilizam testes de estacionariedade em relação ao déficit e à dívida do governo norte-americano. Pastore (1995) e Luporini (2000) analisam a sustentabilidade da política fiscal do governo federal no caso brasileiro. Ao tratar do problema da sustentabilidade da política fiscal do Estado do Rio Grande do Sul, no período de 1970 a 1997, Marques Junior (2005) realiza testes de estacionariedade das relações "dívida mobiliária/PIB”, "déficit primário/ PIB" e "déficit público/PIB" no caso do Estado do Rio Grande do Sul para o período 1970-97 e obtém resultados que indicam a sustentabilidade da política fiscal gaúcha para o período analisado.
Para Hakkio e Rush (1991) e Tanner e Liu (1994), a política fiscal de um governo é sustentável se as variáveis "receita" e "despesa" cointegram. Neste trabalho, considera-se uma política fiscal sustentável quando há cointegração entre as variáveis "receita tributária/produto" e “despesa/produto". Assim, são aplicados testes de cointegração entre as variáveis "despesa total/PIB" e "receita tributária/PIB” para o caso do Rio Grande do Sul. Os testes de cointegração cobrem os períodos de 1970 a 1997 e de 1970 a 2003. Os resultados obtidos para as duas amostras distintas também indicam a sustentabilidade da política fiscal gaúcha no longo prazo.

$\mathrm{O}$ artigo está assim dividido. Na segunda seção, propõe-se o arcabouço teórico e obtém-se, valendo-se de Hakkio e Rush (1991), a regressão básica para os testes de cointegração. $\mathrm{Na}$ terceira seção, é apresentada a análise empírica para o caso do Rio Grande do Sul, e, por fim, na quarta seção, são tecidas as considerações finais.

\section{2_Arcabouço teórico}

Marques Junior (2005), com base em Walsh (1998), obtém, a exemplo de Pas- 
i Para Fischer e Easterly (1990), a hipótese $\mathrm{r}<\lambda$ não é sustentável em longo prazo, já que, à medida que se acumula dívida pública, há pressão sobre o mercado de títulos, o que provoca elevação da taxa de juros e declínio do crescimento econômico. De fato, quando $r<\lambda$, a restrição orçamentária intertemporal não tem limite e, portanto, a questão da sustentabilidade perde sentido. Assim, a hipótese $r>\lambda$ é tida como a mais razoável. Becker e Paalzow (1996) têm a mesma opinião.

\footnotetext{
2 O "jogo não Ponzi" (no Ponzi game) ocorre em situações nas quais o governo não consegue vender os títulos públicos para os agentes econômicos, mesmo pagando taxas de juros elevadas, levando o governo a decretar o default $\mathrm{da}$ dívida pública.
}

tore (1995), Giambiagi e Rigolon (2000) e Luporini (2000), a equação que expressa a dinâmica da razão dívida/produto:

$$
b_{t}-b_{t-1}=g_{t}-\tau_{t}-s_{t}+(r-\lambda) b_{t-1}
$$

Essa equação afirma que a variação na razão dívida/produto, $b_{t}-b_{t-1}$ é igual ao resultado primário do setor público, $g_{t}-\tau_{t}$, menos a senhoriagem, $s_{t}$, e mais o produto entre a razão dívida/produto do período anterior, $\mathrm{b}_{\mathrm{t}-1}$, e a diferença dada pela taxa de juros real ex post $(\mathrm{r})$, e a taxa de crescimento do produto real $(\lambda), r-\lambda$. A equação (1) está expressa em termos reais e ajustada para o crescimento do produto.

Assim, a razão dívida/produto cresce se o déficit primário exceder o montante de senhoriagem e se a taxa de juros real ex post exceder a taxa de crescimento do produto real per capita. A relação dívida/produto decresce se a taxa de crescimento do produto real superar a taxa de juros real ex post. Por último, se $\mathrm{r}<\lambda$, a razão dívida/produto diminui ao longo do tempo, e déficits primários superiores à receita de senhoriagem são sustentáveis. ${ }^{1}$

Supondo-se a receita de senhoriagem igual a zero, a dinâmica da razão dívida/produto é dada por:

$$
b_{t}-b_{t-1}=g_{t}-\tau_{t}+(r-\lambda) b_{t-1}
$$

Assim, se $r>\lambda$ e houver déficits primários, a razão dívida/produto crescerá a uma taxa superior a $\mathrm{r}-\lambda$. Em algum ponto do tempo, o governo não conseguirá rolar a dívida e terá de adotar medidas visando interromper o processo de acumulação de dívida pública. O ponto no qual o processo será interrompido depende das expectativas dos agentes. Quando o público perceber que a política fiscal é insustentável, deixará de comprar títulos públicos, forçando uma mudança de política. Todavia, se $\mathrm{r}>\lambda$ e o déficit primário for igual a zero, a razão dívida/produto crescerá à taxa $\mathrm{r}-\lambda$. Se $\mathrm{r}>\lambda$ e o governo produzir um superávit primário, a razão dívida/produto crescerá a uma taxa inferior a $r-\lambda$. Finalmente, se $\mathrm{r}>\lambda$, e o superávit primário for suficientemente elevado, a razão dívida/ produto poderá permanecer constante ou, até mesmo, decrescer.

Levanta-se, então, a seguinte questão: quando uma política fiscal é sustentável?

Admitindo-se a condição chamada de "jogo não Ponzi”, segundo a qual os credores não vão comprar indefinidamente os títulos públicos, uma política fiscal é sustentável se a dívida em dado período de tempo for igual ao valor presente dos superávits primários futuros. ${ }^{2}$ Em outras palavras, uma política fiscal é 
sustentável se dada sequência de dívida pública e déficits primários satisfazem à condição de equilíbrio orçamentário intertemporal. Choques eventuais podem gerar déficits primários, porém, a fim de que a condição de equilíbrio intertemporal seja satisfeita, necessariamente superávits primários surgirão em períodos posteriores. Uma política fiscal é insustentável caso a condição de equilíbrio orçamentário intertemporal não seja satisfeita.

Pastore (1995) observa que o atendimento da condição de equilíbrio orçamentário intertemporal

[...] não requer que a divida pública convirja para um valor constante, podendo ocorrer mesmo que ela cresça indefinidamente, mas é claro que quaisquer regras fiscais que façam com que a divida tenda para um valor constante conduzem àquele atendimento (Pastore, 1995, p. 185).

Quando a dívida cresce indefinidamente, a razão dívida/produto pode não convergir para um valor constante; nesse caso, a condição de equilíbrio orçamentário intertemporal é atendida, segundo Moraes Junior (2003), desde que a relação dívida/produto cresça a uma taxa inferior à diferença entre a taxa de juros real e a taxa de crescimento do produto real (admitindo-se que a taxa de juros real é maior do que a taxa de crescimento do produto real).

Moraes Junior (2003) chama a atenção para o fato de que a condição de “jogo não Ponzi” é necessária, mas não suficiente para se obter a convergência da relação dívida/produto a um valor constante. Daí alguns autores preferirem a definição de política fiscal sustentável como aquela que implica a convergência da razão dívida/produto a um valor constante.

Hamilton e Flavin (1985), Wilcox (1989), Trehan e Walsh (1991) entendem que a política fiscal de um governo é sustentável se o comportamento das variáveis "déficit público" e "dívida pública" são estacionárias. Para Hakkio e Rush (1991) e Tanner e Liu (1994), a política fiscal de um governo é sustentável se as variáveis "receita" e "despesa" cointegram. Neste trabalho, considera-se uma política fiscal sustentável quando há cointegração entre as variáveis "receita tributária/produto" e "despesa total (incluindo o serviço da dívida pública)/produto”.

Então, supondo-se que a taxa de crescimento do produto real seja zero, de (2) obtém-se a seguinte equação: ${ }^{3}$

$g_{t}+(1+r) b_{t-1}=\tau_{t}+b_{t}$
Note-se que aqui, diferentemente da análise de Hakkio e Rush (1991), as variáveis estão em relação ao produto. 
Resolvendo-se a equação (3) para "frente", chega-se ao seguinte resultado:

$b_{t-1}=\sum_{j=0}^{\infty} \beta^{j+1}\left(\tau_{t+j}-g_{t+j}\right)+\lim _{j \rightarrow \infty} \beta^{j+1} b_{t+j}$

em que $\beta=1 /(1+r)$.

De (3), tem-se

$(1+r) b_{t}-(1+r) b_{t-1}=\tau_{t+1}-\tau_{t}-\left(g_{t+1}-g_{t}\right)+b_{t+1}-b_{t}$.

Substituindo-se sucessivamente $b_{t}$ e $b_{t-1}$ nessa última expressão, obtém-se:

$g_{t}+r b_{t-1}=\tau_{t}+\sum_{j=0}^{\infty} \beta^{j-1}\left(\Delta \tau_{t+j}-\Delta g_{t+j}\right)+\lim _{j \rightarrow \infty} \beta^{j+1} b_{t+j}$

O lado esquerdo de (5) representa os gastos totais do governo em bens e serviços, transferências e pagamento de juros sobre a dívida. Os gastos totais são

${ }_{4}$ Nota-se que se $b>1$, também há garantia de sustentabilidade intertemporal.

5 Para Tanner e Liu (1994), a regressão (7) pode ser interpretada como um teste da restrição orçamentária intertemporal, dadas as seguintes condições: 1) a receita tributária/PIB e a despesa total/PIB seguem um passeio aleatório com drifts, respectivamente, $\alpha_{1} \mathrm{e}$ $\left.\alpha_{2} ; 2\right)$ a taxa de juros real, $r_{t}$, é estacionária com média $\mathrm{r}$. denotados por gg.

Assumindo-se que $\tau$ e g seguem passeios aleatórios com drift, tem-se:

$$
\begin{aligned}
& \tau_{t}=\alpha_{1}+\tau_{t-1}+\varepsilon_{1 t} \\
& g_{t}=\alpha_{2}+g_{t-1}+\varepsilon_{2 t}
\end{aligned}
$$

Neste caso, a equação (5) pode ser reescrita assim:

$$
\begin{aligned}
& g g_{t}=\alpha+\tau_{t}+\lim _{j \rightarrow \infty} \beta^{j+1} b_{t+j}+\varepsilon_{t} \\
& \text { em que } \alpha=\sum \beta^{j-1}\left(\alpha_{1}-\alpha_{2}\right) \text { e } \\
& \varepsilon_{t}=\sum \beta^{j-1}\left(\varepsilon_{1 t}-\varepsilon_{2 t}\right) .
\end{aligned}
$$

Admitindo-se que o termo com limite é igual a zero, ou seja, que se aplica a condição de "jogo não Ponzi", reescreve-se (6) como uma equação de regressão:

$\tau_{\mathrm{t}}=\mathrm{a}+\operatorname{bgg}_{\mathrm{t}}+\varepsilon_{\mathrm{t}}$

A regressão acima é um teste da restrição orçamentária intertemporal na qual a hipótese nula é a de que b seja igual a um e $\varepsilon_{\mathrm{t}}$ estacionário. Em outras palavras, se gg e $\tau_{t}$ são não estacionários, a hipótese nula é a de que $\mathrm{b}=1$ e $\mathrm{gg}_{\mathrm{t}} \mathrm{e} \tau_{\mathrm{t}}$ cointegram. ${ }^{4}$ Quando gg e $\tau$ são não estacionários, a cointegração é uma condição necessária para o governo satisfazer sua restrição orçamentária em valor presente. ${ }^{5}$ Se, por exemplo, $g_{\mathrm{t}}$ é não estacionário enquanto $\tau_{\mathrm{t}}$ é estacionário, não existe relação de longo prazo entre $\mathrm{gg}_{\mathrm{t}} \mathrm{e}$ $\tau_{\mathrm{t}}$. Intuitivamente, isso quer dizer que o governo viola sua restrição orçamentária intertemporal porque gg tende a crescer, o que não ocorre com $\tau$. Nesse caso, o b estimado converge para zero.

A hipótese da sustentabilidade da política fiscal do Rio Grande do Sul é testada por Marques Junior (2005), aplicando-se testes para raiz unitária para as variáveis "dívida mobiliária/PIB", "déficit primário/PIB" e "déficit público/PIB”. 
$\mathrm{Na}$ próxima seção, são aplicados testes de cointegração entre a receita tributá$\mathrm{ria} / \mathrm{PIB}, \tau_{\mathrm{t}}$, e a despesa total/PIB, $\mathrm{gg}_{\mathrm{t}}$. O objetivo é estimar a regressão (7) considerando-se dois períodos 1970 a 1997 e 1970 a 2003.

\section{3_ A política fiscal do Estado do Rio Grande do Sul foi sustentável nos períodos 1970 a 1997 e 1970 a 2003?}

\section{1_ Os dados e uma justificativa}

O objeto de estudo é a Administração Direta do Estado do Rio Grande do Sul. Os dados originais da despesa total e da receita tributária foram obtidos na Secretaria da Fazenda do Estado, mais especificadamente, o Balanço Geral do Estado de diversos anos.

A fonte dos dados do PIB estadual é a Fundação de Economia e Estatística (FEE). É importante ressaltar que a série do PIB estadual de 1970 a 2003 é uma combinação de duas séries: uma que parte de 1970 e vai até 1985 , e outra de 1985 a 2003. Segundo o Núcleo de Contas Regionais da FEE, houve mudança de metodologia no cálculo do PIB. A série do PIB (ver Anexo) foi montada tomando-se as séries de 1970 até 1985 e de 1986 a 2003.
As relações despesa total/PIB, gg, e receita tributária/PIB, $\tau$, foram obtidas após a conversão das variáveis para real $(\mathrm{R} \$)$. A divisão de cada variável em relação ao PIB do período correspondente equivale a usar o mesmo deflator para as séries. Os softwares utilizados foram o Eviews (versão 5.0) e o Stata (versão 10.1).

A escolha de duas amostras, (1970 a 1997) e (1970 a 2003), justifica-se porque os resultados obtidos para o primeiro período podem ser comparados com o estudo de Marques Junior (2005) e porque, ao se aplicarem testes, também para o segundo período tem-se um acréscimo em termos de análise.

\section{2_Cointegração}

Antes de aplicar o teste de cointegração, observa-se no Gráfico 1 a evolução das variáveis gg e $\tau$ ao longo do período de 1970 a 2003, ambas medidas em termos nominais e em relação ao PIB. Note-se que, de 1970 a 1980, tanto gg como $\tau$ apresentaram tendência de queda, comportamento que se reverteu no período de 1981 a 1994, quando gg cresceu mais do que $\tau$; de 1995 a 1997, aumentou a diferença entre gg e $\tau$; porém, de 1998 a 2003, tal distância vem caindo, seja porque gg vem se reduzindo, seja porque $\tau$ retomou uma trajetória de crescimento. 
Gráfico 1_Despesa total e receita tributária em relação ao PIB (Administração Direta) do Estado do Rio Grande do Sul (1970-2003)

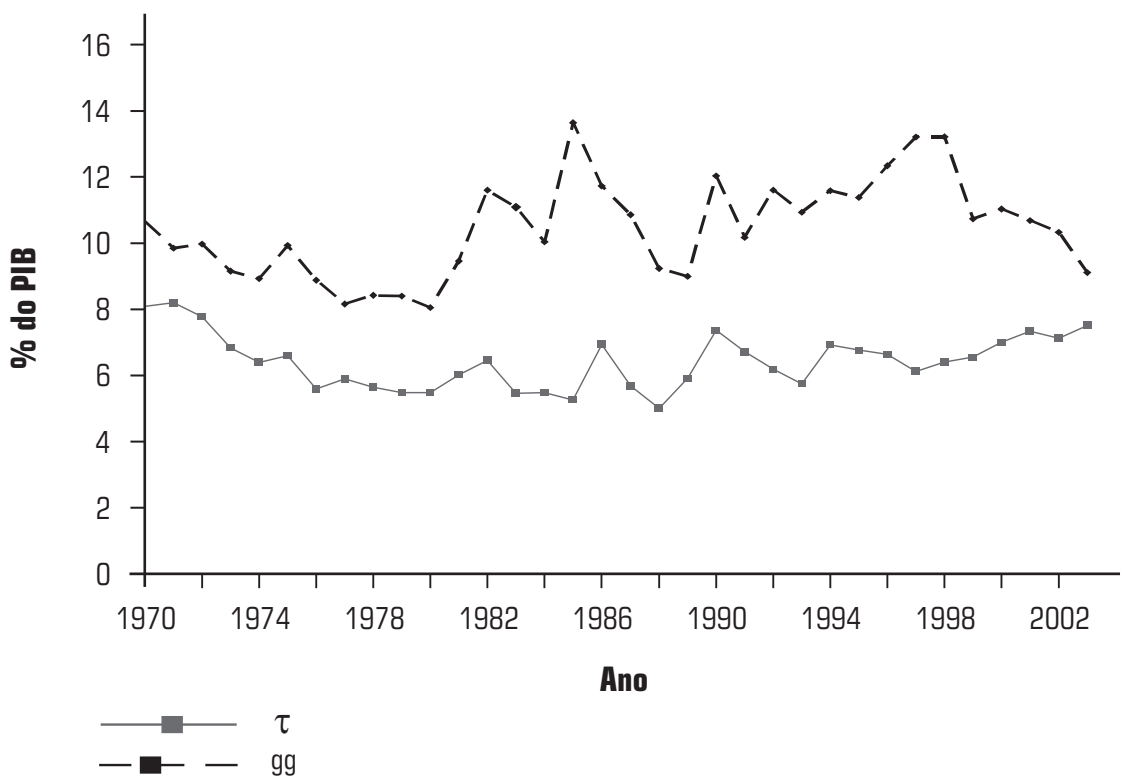

Fonte: Tabelas 1 e 2 no Anexo

Não cabe aqui retomar os pormenores da trajetória fiscal do Estado do Rio Grande do Sul, apenas se observa que, entre 1994 e 1997, a situação das finanças públicas estadual agravou-se em função da deterioração da situação fiscal e de baixa taxa média de crescimento do produto real no período. A situação fiscal decaiu em função de uma política fiscal "expansionista" colocada em prática no governo Britto (1995-1998) e da estabilidade dos preços (este fator teve como implicação a não diminuição do valor real das despesas públicas em atraso).

Diante da deterioração das contas públicas, o Estado viu-se obrigado a assinar, em 1998, um acordo com o governo federal de renegociação da dívida estadual. No bojo desse acordo, o Estado assumiu o compromisso com um ajuste fiscal rigoroso, através do estabelecimento de metas de superávit primário, aumento da arrecadação e controle do endividamento público. Portanto, no pe- 
ríodo de 1998 a 2003, houve mudança da política fiscal estadual, tendo-se a queda da relação DT/PIB e a elevação da carga tributária estadual. ${ }^{6}$

A metodologia para o teste de cointegração é a de Engle e Granger. ${ }^{7} \mathrm{O}$ primeiro passo é verificar se as variáveis são estacionárias e determinar a ordem de integração de cada uma. Existe grande variedade de testes de raiz unitária na literatura econômica, entre os quais se destaca o teste de Dickey-Fuller Aumentado (ADF). Por tratar-se de um teste de baixa potência, ou seja, tende a aceitar a hipótese nula mesmo quando essa é falsa, são utilizados também os testes de DickeyFuller-GLS (DF-GLS) e Ng-Perron com o objetivo de amenizar o problema e tornar os resultados mais robustos. ${ }^{8}$

Para a escolha do número ótimo de defasagens, $\mathrm{p}^{*}$, seguiu-se o seguinte procedimento: a partir de um número de defasagens igual a cinco $(p=5)$, as defasagens estatisticamente significativas foram selecionadas; a defasagem ótima, $\mathrm{p}^{*}$, foi determinada utilizando-se dos critérios Akaike (AIC) e Schwarz (SBC) no caso do teste ADF. Para os testes DF-GLS e Ng-Perron, a defasagem ótima, $\mathrm{p}^{*}$, foi determinada utilizando-se do critério de informação Akaike modificado (MAIC), sugerido por Ng e Perron (2001). ${ }^{\text {? }}$
Os resultados dos testes para raiz unitária de gg e $\tau$ para o período 1970 a 2003 estão apresentados na Tabela 1. Observa-se que o teste ADF falhou em rejeitar a hipótese nula de raiz unitária para as variáveis e, portanto, elas são não estacionárias. As estatísticas calculadas e Shin (1992), conhecido pela sigla KPSS, é adotado em alguns artigos porque a hipótese de estacionariedade é testada diretamente. A hipótese nula do teste KPSS assume que a série é estacionária, por isso é utilizado, geralmente, com a finalidade de uma análise comprobatória. Esse teste, porém, também sofre do problema de baixa potência. Segundo Maddala e Kim (1998), não há consenso sobre o uso do teste KPSS como uma análise comprobatória, uma vez que os resultados encontrados na literatura para algumas séries se mostram contraditórios.

9 Cabe observar que os testes de raiz unitária são sensíveis ao número de defasagens usado, por isso busca-se escolher o número ótimo de forma a não penalizar o poder do teste. Conforme Maddala e Kim (1998), a diferença no número de defasagens não altera se o processo é I(1) ou I(0), mas sim o tamanho e o poder do teste. 


\footnotetext{
10 Esse resultado pode estar relacionado a problemas oriundos do uso de pequenas amostras como é o caso do presente artigo. No caso específico de pequenas amostras, o teste ADF é mais robusto, ou seja, menos sujeito às distorções de tamanho.
}

Tabela 1_Testes para raiz unitária para gg e $\tau$ (1970-2003)

\begin{tabular}{|c|c|c|c|c|c|c|c|c|}
\hline \multirow{2}{*}{ Variável } & \multicolumn{2}{|c|}{ ADF $^{1}$} & \multicolumn{2}{|c|}{ DF-GLS } & \multicolumn{4}{|c|}{ Ng-Perron ${ }^{2}$} \\
\hline & estat. & $\mathbf{p}^{*}$ & estat. & $\mathbf{p}^{*}$ & $\mathbf{M z}_{\alpha}$ & $\mathbf{p}^{*}$ & $\mathrm{Mz}_{\mathrm{t}}$ & $\mathbf{p}^{*}$ \\
\hline $\mathrm{gg}_{\mathrm{t}}$ & $-3,0963$ & 0 & $-3,0778$ & 0 & $-11,9076$ & 0 & $-2,3334$ & 0 \\
\hline$\tau_{t}$ & $-3,3390$ & 1 & $-0,9670$ & 3 & $-1,4186$ & 3 & $-0,6673$ & 3 \\
\hline$\Delta g_{\mathrm{t}}$ & $-7,3066 * * *$ & 0 & $-6,7984 * * *$ & 0 & $-14,1629 * * *$ & 0 & $-2,6432 * * *$ & 1 \\
\hline$\Delta \tau_{\mathrm{t}}$ & $-5,7168 * * *$ & 2 & $-5,6356 * * *$ & 2 & $-3.581,88^{* * *}$ & 2 & $-42,3195^{* * *}$ & 1 \\
\hline
\end{tabular}

1) As estatísticas foram confrontadas com os valores críticos de MacKinnon;

2) As estatísticas foram comparadas com os valores críticos tabulados por Elliot, Rothenberg e Stock (1996) e por Ng e Perron (2001). $\left.{ }^{* * *}\right)$ rejeita-se a hipótese da existência de raiz unitária a 1\% de significância.

Fonte: Cálculo dos autores a partir das informações da Fundação de Economia e Estatística (FEE) e da Secretaria da Fazenda do Estado do RS.

Nota: As estatísticas dos testes ADF, DF-GLS e Ng-Perron sobre gg e $\tau$ consideram o modelo com tendência e constante. As estatísticas dos testes ADF, DF-GLS e Ng-Perron sobre $\Delta$ gg e $\Delta \tau$ consideram o modelo sem tendência e com constante.

para os testes DF-GLS e Ng-Perron também indicam a presença de raiz unitária nas séries.

Para verificar se as variáveis são integradas de ordem 1, I(1), obteve-se a primeira diferença das variáveis e testouse a existência de raiz unitária. Os resultados dos testes ADF, DF-GLS e Ng-Perron indicam que a hipótese nula de não estacionariedade é rejeitada e mostra que ambas as variáveis são I(1).

Na Tabela 2, têm-se os mesmos testes, porém considerando-se o período 1970 a 1997. Observa-se que gg e $\tau$ são não estacionárias aos três níveis de significância, exceto para $\tau$ no teste DF-GLS ao nível de significância de $5 \% .{ }^{10}$ Igualmente aos resultados apresentados na
Tabela 1, após a primeira diferença, as variáveis se tornam estacionárias e, portanto, ambas são de ordem I(1).

As séries gg e $\tau$ estão sujeitas aos mais diversos tipos de choque e a impactos de diferentes tamanhos. Por exemplo, entre 1979 e 1984, em decorrência da Segunda Crise do Petróleo, houve forte elevação das taxas de juros mundiais, o que provocou abrupta elevação dos juros domésticos e, por conseguinte, das despesas com o serviço da dívida pública estadual. Tal elevação das despesas com os juros teve forte impacto sobre a trajetória da variável gg. Assim, suspeitase que o referido choque exógeno possa representar quebra estrutural na série da variável gg. 
Tabela 2_ Testes para raiz unitária para gg e $\tau$ (1970-1997)

\begin{tabular}{|c|c|c|c|c|c|c|c|c|}
\hline \multirow{2}{*}{ Variável } & \multicolumn{2}{|l|}{ ADF 1} & \multicolumn{2}{|l|}{ DF-GLS' } & \multicolumn{4}{|c|}{ Ng-Perron ${ }^{2}$} \\
\hline & estat. & $\mathbf{p}^{*}$ & estat. & $\mathbf{p}^{*}$ & $\mathbf{M z}$ & $\mathbf{p}^{*}$ & $\mathrm{Mz}_{\mathrm{t}}$ & $\mathbf{p}^{\prime \prime}$ \\
\hline$g g_{\mathrm{t}}$ & $-3,3524$ & 3 & $-1,0429$ & 3 & $-1,8681$ & 3 & $-0,9509$ & 3 \\
\hline$\tau_{t}$ & $-3,4572$ & 0 & $-3,4342^{* *}$ & 0 & $-10,8786$ & 0 & $-2,2670$ & 0 \\
\hline$\Delta \mathrm{gg}_{\mathrm{t}}$ & $-5,4415^{* * *}$ & 2 & $-5,5394 * * *$ & 2 & $-925,069 * * *$ & 2 & $-21,5043$ & 2 \\
\hline $\begin{array}{c}\Delta \tau_{\mathrm{t}} \\
\ldots \ldots\end{array}$ & $\begin{array}{c}-6,9896 * * * \\
\ldots, \ldots \ldots \ldots \ldots\end{array}$ & $\begin{array}{l}0 \\
0 .\end{array}$ & $\begin{array}{r}-6,8323 * * * \\
\ldots \ldots \ldots \ldots \ldots\end{array}$ & $\begin{array}{l}0 \\
0 .\end{array}$ & $\begin{array}{c}11,6086 * * * \\
\ldots \ldots \ldots \ldots \ldots \ldots\end{array}$ & $\begin{array}{l}0 \\
0\end{array}$ & $\begin{array}{l}-2,3842 \\
\ldots \ldots \ldots \ldots\end{array}$ & $\begin{array}{l}0 \\
0\end{array}$ \\
\hline
\end{tabular}

1) As estatísticas foram confrontadas com os valores críticos de MacKinnon.

2) As estatísticas foram comparadas com os valores críticos tabulados por Elliot, Rothenberg e Stock (1996) e por Ng e Perron (2001). $(* * *)$ rejeita-se a hipótese da existência de raiz unitária a $1 \%$ de significância.

Fonte: Cálculo dos autores a partir das informações da Fundação de Economia e Estatística (FEE) e da Secretaria da Fazenda do Estado do RS.

Nota: As estatísticas estimadas dos testes ADF, DF-GLS e Ng-Perron sobre gg e $\tau$ consideram o modelo com tendência e constante. As estatísticas dos testes ADF, DF-GLS e Ng-Perron sobre $\Delta g g$ e $\Delta \tau$ consideram o modelo sem tendência e com constante.

Ao longo do período em análise, ocorreram diversas mudanças institucionais. A mais importante foi a promulgação da Constituição Federal de 1988, na qual se estabeleceu um novo sistema tributário e ampliaram-se os gastos sociais para os três níveis de governo. Dessa forma, suspeita-se que tal mudança possa ter alterado as trajetórias das variáveis gg e $\tau$. A assinatura do acordo de renegociação da dívida estadual com a União em 1998 e a Lei de Responsabilidade em 2000 são outros exemplos de mudanças institucionais que podem ter afetado as trajetórias das duas variáveis. No acordo de renegociação da dívida pública, o Estado se comprometeu a diminuir a relação dívida/PIB estadual através do controle de gastos e elevação da arrecadação de impostos. A Lei de Responsabilidade Fiscal em 2000 estabeleceu um conjunto de regras de finanças públicas a serem seguidas pelos três níveis de governo.

Portanto, é intuitivo se pensar que essas mudanças devem ser levadas em conta tanto nos testes de raiz unitária. Perron (1993), Zivot e Andrews (1992) e Perron e Vogelsang (1993) apresentam testes de raiz unitária quando o período da quebra estrutural é desconhecido. Para tanto, desenvolvem metodologias para determinar endogenamente o período da quebra. Lembra-se de que a quebra estrutural é um evento que gera efeitos sobre o comportamento da série e pode ser modelada de diferentes maneiras.

Ao se considerar a possibilidade de duas quebras estruturais, apresentam- 
11 Para mais detalhes sobre a classificação das quebras, ver Perron (1993) e Perron e Vogelsang (1993). O modelo proposto por Clemente et al. (1998) com duas quebras para o caso IO é o seguinte:

$$
\begin{aligned}
y_{t} & =\mu+\rho y_{t-1}+\delta_{1} D T B_{1 t}+ \\
& +\delta_{2} D T B_{2 t}+d_{1} D U_{1 t}+ \\
& +d_{2} D U_{2 t}+\sum_{i=1}^{k} c_{i} \Delta y_{t-i}+e_{t},
\end{aligned}
$$

em que DTB it $_{\text {é uma variável }}$ dummy crash que assume valor igual a 1 se $\mathrm{t}=\mathrm{TB}_{\mathrm{i}}+1$

(i $=1,2)$ e 0 caso contrário; $\mathrm{DU}_{\text {it }}$ é uma variável dummy intercepto que assume valor igual a 1 se $\mathrm{t}>\mathrm{TB}_{\mathrm{i}}(\mathrm{i}=1,2) \mathrm{e}$ 0 caso contrário; e $\mathrm{TB}_{1}$ e $\mathrm{TB}_{2}$ são os períodos das quebras estruturais. Para o caso AO, os modelos são os seguintes:

$$
\begin{aligned}
y_{t} & =\mu+d_{1} D U_{1 t}+d_{2} D U_{2 t}+\tilde{y}_{t} \\
\tilde{y}_{t} & =\sum_{i=0}^{k} \omega_{1 i} D T B_{1 t-i} .+ \\
& +\sum_{i=0}^{k} \omega_{2 i} D T B_{2 t-i}+\rho \tilde{y}_{t-1}+ \\
& +\sum_{i=1}^{k} c_{i} \Delta \tilde{y}_{t-i}+e_{t} .
\end{aligned}
$$

se na Tabela 3 os testes de raiz unitária propostos por Clemente et al. (1998). Os autores classificam as quebras estruturais na média de uma série em dois tipos de outlier: o outlier aditivo (additive outlier, AO) e o outlier inovacional (innovational outlier, IO). O modelo AO admite a situação de uma mudança abrupta na média. O modelo IO leva em conta o caso de mudanças graduais na média. ${ }^{11}$

Os testes para raiz unitária com as quebras indicam que as séries não são estacionárias e que as quebras são signifi-

\begin{tabular}{|c|c|c|c|c|c|c|c|c|}
\hline Variável & $\begin{array}{l}\text { Tipo de } \\
\text { Outlier }\end{array}$ & $\hat{\mu}$ & $\hat{\mathbf{d}}_{1}$ & $\mathbf{t}_{\hat{d}_{1}}$ & TB & $\hat{\rho}$ & $\min \mathbf{t}_{\hat{\mathbf{p}}}$ & $\begin{array}{c}\text { Valores críticos } \\
\text { para min } \mathbf{t}_{\hat{\mathbf{p}}}\end{array}$ \\
\hline$g g_{t}$ & $\mathrm{AO}$ & 9.46 & 1.67 & 3.86 & 83 & -0.69 & -3.41 & -3.560 \\
\hline$\tau_{t}$ & $\mathrm{AO}$ & 6.28 & 0.40 & 1.39 & - & -0.45 & -3.44 & -3.560 \\
\hline$g g_{t}$ & $\mathrm{IO}$ & 6.28 & 1.62 & 2.97 & 80 & -0.70 & -3.98 & -4.270 \\
\hline$\tau_{t}$ & $\mathrm{IO}$ & 4.03 & 0.83 & 4.36 & 88 & -0.71 & -3.95 & -4.270 \\
\hline$\Delta g g_{\mathrm{t}}$ & IO & -0.08 & -0.10 & -0.21 & 87 & -1.31 & -7.44 & -4.270 \\
\hline$\Delta \tau_{\mathrm{t}}$ & $\mathrm{IO}$ & -0.36 & 0.53 & 2.34 & 85 & -2.26 & -6.35 & -4.270 \\
\hline
\end{tabular}
cativas. Como se suspeitava, a série da variável $\tau$ apresentou quebra gradual na média a partir de 1988, do tipo IO, coincidindo com o ano de promulgação da Constituição Federal. Para a série da variável gg, têm-se duas mudanças estru-

\section{Tabela 3_ Testes de raiz unitária com duas quebras (amostra 1970-2003)}

Fonte: Cálculo dos autores a partir das informações da Fundação de Economia e Estatística (FEE) e da Secretaria da Fazenda do Estado do RS.

Nota: As estimativas têm por base os modelos propostos na nota de roda pé 9 com uma quebra apenas. Nos testes com duas quebras, apenas uma se mostrou significativa. O valor de $\mathrm{k}$ foi selecionado, seguindo-se os procedimentos sugeridos por Perron $\mathrm{e}$ Vogelsang (1992). Os valores críticos foram tabulados por Clemente et al. (1998). 
A Tabela 4 apresenta os resultados das estimações para as duas amostras.

Dada a identificação das quebras estruturais, incorporaram-se ao modelo (7) três variáveis dummy. A variável dummy Q79 assume valor igual a 1 de 1979 a 1988 e zero nos demais períodos. Portanto, visa captar a quebra estrutural causada pelo Segundo Choque do Petróleo. A variável dummy Q88 assume valor igual a 1 de 1988, ano da promulgação da nova Constituição Federal, a 1997 e zero nos demais períodos. A terceira variável dummy Q98 é igual a 1 de 1998 a 2003 e zero nos demais períodos. Tal variável visa captar as mudanças que surgem a partir do plano de renegociação da dívida estadual com a União assinado em abril de 1998. A inclusão das variáveis dummy visa ao não comprometimento das propriedades dos estimadores.

Examinam-se, então, os resíduos estimados de cada regressão a fim de constatar a existência ou não de raiz unitária. Para tanto, considere a autorregressão dos resíduos:

$$
\Delta \hat{e}_{t}=a_{1} \hat{e}_{t-1}+\varepsilon_{t}
$$

A Tabela 5 apresenta os resultados das estimações para as duas amostras.

\section{Tahela 4_Testes de cointegração}

\begin{tabular}{|c|c|c|}
\hline & Amostra 1970-2003 & Amostra 1970-1997 \\
\hline & Variável dependente: $\tau$ & Variável dependente: $\tau$ \\
\hline a & $\begin{array}{c}4.91 \\
(0.0000)\end{array}$ & $\begin{array}{c}4.44 \\
(0.0002)\end{array}$ \\
\hline $\mathrm{b}$ & $\begin{array}{c}0.19 \\
(0.0385)\end{array}$ & $\begin{array}{c}0.25 \\
(0.0246)\end{array}$ \\
\hline Q79 & $\begin{array}{c}-1.21 \\
(0.0012)\end{array}$ & $\begin{array}{c}-1.27 \\
(0.0016)\end{array}$ \\
\hline Q88 & $\begin{array}{c}-0.86 \\
(0.0218) \\
\end{array}$ & $\begin{array}{c}-0.89 \\
(0.0263)\end{array}$ \\
\hline Q98 & $\begin{array}{c}0.18 \\
(0.0144)\end{array}$ & - \\
\hline $\mathrm{R}^{2}$ & 0,41 & 0,37 \\
\hline
\end{tabular}

Fonte: Cálculo dos autores a partir das informações da Fundação de Economia e Estatística (FEE) e da Secretaria da Fazenda do Estado do RS.

Nota: valor- $p$ entre parêntesis. 
Tabela 5_Testes de estacionariedade dos resíduos

\begin{tabular}{c} 
Amostra 1970-2003 \\
Variável dependente $\Delta \hat{\mathbf{e}}_{\mathrm{t}-1}$ \\
\hline
\end{tabular}

${ }^{(*)}$ Rejeita-se a hipótese de raiz unitária a $5 \%$ de significância segundo os valores críticos tabelados por Mackinnon.

Fonte: Cálculo dos autores a partir das informações da Fundação de Economia e

Estatística (FEE) e da Secretaria da Fazenda do Estado do RS

Nota: estatísticas- $t$ entre parêntesis.

Da Tabela 5, pode-se rejeitar a hipótese nula de que $\mathrm{a}_{1}$ é igual a zero para as duas amostras. Então, conclui-se que os resíduos não contêm uma raiz unitária, ou seja, são estacionários. Assim, pode-se aceitar que as variáveis $\mathrm{gg}_{\mathrm{t}}$ e $\tau_{\mathrm{t}}$ cointegram. Dado que as séries $g_{\mathrm{t}} \mathrm{e} \tau_{\mathrm{t}}$ são de ordem I(1), e os resíduos das regressões são estacionários, conclui-se que as séries são cointegradas de ordem $(1,1), \mathrm{CI}(1,1)$.

Em suma, os testes de cointegração deram suporte à hipótese de sustentabilidade da política do Estado do Rio Grande do Sul não só para o período 1970-1997, como também para o período 1970-2003.

\section{Considerações finais}

Hamilton e Flavin (1985) e Wilcox (1989) têm a convicção de que a política fiscal de um governo é sustentável se o déficit público e a dívida pública seguem processos estocásticos estacionários. Aplicando-se testes para raiz unitária para as variáveis "dívida mobiliária/PIB", "déficit primário/PIB" e "déficit público/ PIB" com os dados do Rio Grande do Sul de 1970 a 1997, Marques Junior (2005) não rejeitou a hipótese de sustentabilidade. Trehan e Walsh (1991), Hakkio e Rush (1991) e Tanner e Liu (1994) definem que uma política fiscal é sustentável se houver presença de cointegração entre as variáveis "receita" e "despesa" do governo. Neste trabalho, uma política fiscal é sustentável quando há cointegração entre as variáveis "receita tributária/PIB" e "despesa total/PIB".

Ressalvando-se o reduzido número de observações e tendo-se em conta os dados disponíveis (nominais e anuais), os resultados dos testes de cointegração não rejeitaram a hipótese de sustentabilidade da política fiscal do Rio Gran- 
de do Sul para os períodos (1970-1997) e (1970-2003). Isto é, existe relação de longo prazo entre as variáveis gg e $\tau$. Intuitivamente, a restrição orçamentária intertemporal do governo gaúcho não foi violada, seja porque gg não apresentou tendência crescente enquanto $\tau$ se manteve constante, seja porque gg não cresceu mais do que $\tau$, ano após ano, nas duas amostras consideradas.

Apesar de a política fiscal do Estado parecer sustentável no longo prazo, o Estado do Rio Grande do Sul está sujeito a enfrentar problemas de financiamento no curtíssimo prazo, o que sugere a necessidade de se manter a trajetória que se iniciou em 1998 de redução da relação despesa total/PIB e de elevação da receita tributária/PIB ou de uma trajetória de crescimento da relação receita tributária/PIB superior (ou igual) ao crescimento da relação despesa total/PIB.

$\mathrm{O}$ estudo proposto não esgota a discussão sobre a sustentabilidade da política fiscal gaúcha. Outros modelos e novos testes com outras variáveis podem ser objetos de análises futuras. 
Referências bibliográficas

\author{
ALMEIDA, Anna Ozorio de. \\ Evolução e crise da divida pública \\ estadual. IPEA, nov. 1996. \\ (Texto para Discussão, 448). \\ BECKER, Torbjörn; PAALZOW, \\ Anders. Real effects of budget \\ deficits: theory and evidence. \\ Swedish Economic Policy Review, \\ n. 2, p. 343-383, 1996. \\ CALAZANS, Roberto \\ B.; BRUNET, Júlio F. G.; \\ MARQUES JR., Liderau dos \\ Santos. Ajuste fiscal: modelos \\ dinâmicos e aplicação para o \\ caso do RS. In: Finanças Públicas: \\ IV Prêmio Tesouro Nacional \\ (coletânea de monografias). \\ Brasília: ESAF, 2000.
}

BEVILAQUA, Afonso S. StateGovernment bailouts in Brazil. Departamento de Economia da PUC-Rio. Draft, maio 1999.

\section{BOTELHO, Ricardo.}

Determinantes do ajuste fiscal dos estados brasileiros. In: Financas Públicas: IV Prêmio Tesouro Nacional (coletânea de monografias). Brasília: ESAF, 2002.

BORDIN, Luis Carlos Vitali. A dívida pública do Rio Grande do Sul: Administração Direta (19511984). In: LAGEMANN, Eugenio (Org.). Rio Grande do Sul 150 anos de finanças públicas. Porto Alegre: FEE, 1985.
CALANDRO, Maria Lucrecia. In: LAGEMANN, Eugenio (Org.). Rio Grande do Sul 150 anos de finanças públicas. Porto Alegre: FEE, 1985.

\section{CLEMENTE, Jesús;}

MONTAÑÉS, Antonio; REYES, Marcelo. Testing for a unit root in variables with a double change in the mean. Economics Letters, 59, p. 175-182, 1998.

ELLIOT, G.; ROTHENBER, T. J.; STOCK, J. H. Efficient Tests for an Autoregressive Unit Root. Econometrica, 64, p. 813-836, 1996.

ENDERS, Walter. Applied econometric time series. Second Edition. John Wiley \& Sons, Inc. 2004

FISCHER, Stanley; EASTERLY, William. The economics of the government budget constraint. The World Bank Research Observer, v. 5, n. 2, p. 127-142, 1990.
GIAMBIAGI, Fábio; RIGOLON, Francisco. O ajuste fiscal de médio prazo: o que vai acontecer quando as receitas extraordinárias acabarem? In: Finanças Públicas: IV Prêmio Tesouro Nacional (coletânea de monografias). Brasília: ESAF, 2000.

HAKKIO, Craig S.; RUSH, Mark. Is the budget deficit "too large?". Economic Inquiry, v. 29, p. 429-445, July 1991.

HAMILTON, James D.; FLAVIN, Marjorie A. On the limitations of government borrowing: a framework for empirical testing. Cambridge: National Bureau of Economic Research, June 1985. (Working Paper, 1632).

KWIATKOWSKI D.; PHILLIPS, P.; SCHMIDT, P.; SHIN, Y.

Testing the null hypothesis of stationarity against the alternative of a unit root: how sure are we that economic time series have a unit root? Journal of Econometrics, v. 54, p. 159-178, 1992.

LUPORINI, Viviane. Sustainability of the brazilian fiscal policy and Central Bank Independence. Revista Brasileira de Economia, v. 54, n. 2, p. 201-226, jan./mar. 2000.
MADDALA, G. S.; KIM, In-Moo. Unit roots, cointegration, and structural change. Cambridge University Press, 1998.

MARQUES JUNIOR, Liderau dos Santos. A sustentabilidade da política fiscal do RS (1970-1997). Ensaios FEE, v. 26, p. 249-270, maio 2005. (n. especial).

MORAES JUNIOR, Aod Cunha de. Ensaios sobre fragmentacãa de governo e ajustamento fiscal. 2003. Tese (Doutorado em Economia). Faculdade de Ciências Econômicas, Universidade Federal do Rio Grande do Sul, Porto Alegre, 2003.

MOURA NETO, Bolívar T.; VIEIRA, Jorge Blascoviscki. Finanças estaduais em 1991: o ajuste ortodoxo frente à crise. Indicadores Econômicos, v. 20, n. 1, maio 1992.

NG, Serena; PERRON, Piere. Lag lenght selection and the construction of unit root test with good size and power. Econometrica, v. 69, n. 6, p. 1519-1554, 2001. 
PASTORE, Affonso Celso. Déficit público, a sustentabilidade do crescimento das dívidas interna e externa, senhoriagem e inflação: uma análise do regime monetário brasileiro. Revista de Econometria, v. 14, n. 2, p. 177-234, nov. 1994/ dez. 1995, 1995.

PERRON, Pierre. Trend, unit root and structural change in macroeconomic time series. In. RAO, B. B. (Ed.). Cointegration: expository essays for the applied economist. Macmillan. 1993.

\section{PERRON, P.; VOGELSANG,}

T. J. A note on the asymptotic distributions of unit root test in the additive outlier model with breaks. Revista de Econometria, v. 13, p. 181-201, 1993.

RIGOLON, Francisco; GIAMBIAGI, Fabio. A renegociação das dívidas e o regime fiscal dos Estados. In: GIAMBIAGI, Fabio; MOREIRA, Mauricio Mesquita (Org.). A economia brasileira nos anos 90. Rio de Janeiro: BNDES, 1999.

RIO GRANDE DO SUL. Finanças do Estado. Porto Alegre: Secretaria da Fazenda do Estado do Rio Grande do Sul. v. 34, 35 e 41. [s.d.].

RIO GRANDE DO SUL. Balanç geral do Estado do Rio Grande do Sul [dados informativos on-line]. Porto Alegre, 2003. Disponível em: <http://www.sefaz.rs.gov. br>. Acesso em: 10 jun. 2004.
RIO GRANDE DO SUL. Finanças públicas [on-line]. Porto Alegre, 2003. Disponível em: < http:// www.sefaz.rs.gov.br $>$. Acesso em: 17 ago. 2004.

RÜCKERT, Isabel Noemia; BORSATTO, Maria Luiza; RABELO, Mercedes. Os desajustes estruturais das finanças públicas do RS nos anos 90. In: FLIGENSPAN, Flávio Benevett (Coord.). A economia gaúcha e reestruturacão nos anos 90.

Porto Alegre: FEE, 2000.

SANTOS, Darcy F. C. dos; CALAZANS, Roberto B. A crise da divida pública do RS - Fundamentos, evolução e perspectivas/19701998. Porto Alegre: Assembleia Legislativa do Estado do Rio Grande do Sul (Comissão de Finanças e Planejamento), nov. 1999.

TANNER, Evan; LIU, Peter. Is the budget deficit "too large"? Some further evidence. Economic Inquiry, p. 511-518, July 1994.

TREHAN, Bharat; WALSH, Carl E. Testing intertemporal budget constraints: theory and applications to U.S. federal budget and current account deficits. Journal of Money, Credit, and Banking, v. 23, n. 2, maio 1991.

WALSH, Carl E. Monetary Theory and Policy. Cambridge: The MIT Press, 1998.
WILCOX, David W. The sustainability of government deficits: implications of the present-value borrowing constraint. Journal of Money, Credit, and Banking, v. 21, n. 3, ago. 1989.

ZIVOT, E.; ANDREWS, D. W. K. Further evidence on the Great Crash, the Oil-Price Shock, and the Unit Root Hypothesis. Journal of Business and Economic Statistics, v. 10, p. 251-270, 1992.

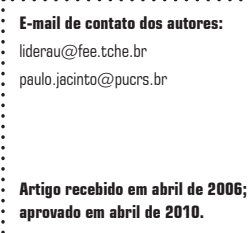


Anexo

\begin{tabular}{|c|c|c|c|}
\hline Anos & Receita Tributária' (R\$) & $\mathrm{PIB}^{2}$ (R\$) & Carga Tributária $=\tau_{t}(\%)$ \\
\hline 1970 & 0,0004293582 & 0,0053127273 & 8,08 \\
\hline 1971 & 0,0005922825 & 0,0072254545 & 8,20 \\
\hline 1972 & 0,0007303844 & 0,0094036364 & 7,77 \\
\hline 1973 & 0,0009963447 & 0,0146036364 & 6,82 \\
\hline 1974 & 0,0013302593 & 0,0208254545 & 6,39 \\
\hline 1975 & 0,0019207102 & 0,0291345455 & 6,59 \\
\hline 1976 & 0,0025416233 & 0,0454654545 & 5,59 \\
\hline 1977 & 0,0040293313 & 0,0683200000 & 5,90 \\
\hline 1978 & 0,0055776222 & 0,0989090909 & 5,64 \\
\hline 1979 & 0,0087595018 & 0,1600290909 & 5,47 \\
\hline 1980 & 0,0187360804 & 0,3426581818 & 5,47 \\
\hline 1981 & 0,0403135880 & 0,6697418182 & 6,02 \\
\hline 1982 & 0,0834082844 & 1,2926581818 & 6,45 \\
\hline 1983 & 0,1864809782 & 3,4141490909 & 5,46 \\
\hline 1984 & 0,6008701935 & 10,9621672727 & 5,48 \\
\hline 1985 & 2 & 38 & 5,26 \\
\hline 1986 & 7 & 101 & 6,93 \\
\hline 1987 & 18 & 317 & 5,68 \\
\hline 1988 & 123 & 2.462 & 5,00 \\
\hline 1989 & 2.223 & 37.598 & 5,91 \\
\hline 1990 & 69.117 & $939.363,40$ & 7,36 \\
\hline 1991 & 313.778 & $4.666 .959,02$ & 6,72 \\
\hline 1992 & 3.403 .833 & $54.964 .960,58$ & 6,19 \\
\hline 1993 & 72.389 .398 & 1.260 .808 .219 & 5,74 \\
\hline 1994 & 2.155 .483 .151 & 31.129 .234 .457 & 6,92 \\
\hline 1995 & 3.631 .675 .187 & 53.652 .946 .828 & 6,77 \\
\hline 1996 & 4.194 .757 .608 & 63.262 .677 .227 & 6,63 \\
\hline 1997 & 4.237 .215 .255 & 69.221 .313 .934 & 6,12 \\
\hline 1998 & 4.515 .641 .472 & 70.541 .889 .405 & 6,40 \\
\hline 1999 & 4.941 .505 .295 & 75.450 .458 .225 & 6,55 \\
\hline 2000 & 5.960 .206 .517 & 85.137 .542 .554 & 7,00 \\
\hline 2001 & 7.138 .685 .853 & 97.310 .194 .511 & 7,34 \\
\hline 2002 & 7.808 .523 .095 & 109.742 .129 .654 & 7,12 \\
\hline 2003 & 9.818 .893 .706 & 130.744 .187 .478 & 7,51 \\
\hline
\end{tabular}

Fontes: 1) Finanças do Estado e Finanças Públicas on-line. Porto Alegre: Secretaria da Fazenda. 2) FEE (Núcleo de Contas Regionais).

Notas: 1) os dados do PIB de 2000 a 2003 são preliminares; 2) a preços correntes. 
Tabela 2_Relação DT/PIB da Administração Direta (1970-2003)

\begin{tabular}{|c|c|c|}
\hline Anos & $\mathrm{DT}^{1}$ (R\$) & $D T / P I B=g g_{t}(\%)$ \\
\hline 1970 & 0,000565925 & 10,65 \\
\hline 1971 & 0,000711944 & 9,85 \\
\hline 1972 & 0,000937672 & 9,97 \\
\hline 1973 & 0,001335484 & 9,14 \\
\hline 1974 & 0,001860253 & 8,93 \\
\hline 1975 & 0,002893229 & 9,93 \\
\hline 1976 & 0,004035937 & 8,88 \\
\hline 1977 & 0,005575929 & 8,16 \\
\hline 1978 & 0,008330993 & 8,42 \\
\hline 1979 & 0,013449504 & 8,40 \\
\hline 1980 & 0,027583114 & 8,04 \\
\hline 1981 & 0,063326955 & 9,45 \\
\hline 1982 & 0,149651869 & 11,60 \\
\hline 1983 & 0,378290693 & 11,09 \\
\hline 1984 & 1,10 & 10,05 \\
\hline 1985 & 5,24 & 13,65 \\
\hline 1986 & 11,82 & 11,73 \\
\hline 1987 & 34,36 & 10,86 \\
\hline 1988 & 227,24 & 9,23 \\
\hline 1989 & $3.381,70$ & 8,99 \\
\hline 1990 & $112.959,97$ & 12,02 \\
\hline 1991 & $474.847,30$ & 10,17 \\
\hline 1992 & $6.376 .873,02$ & 11,60 \\
\hline 1993 & $137.868 .403,92$ & 10,93 \\
\hline 1994 & $3.608 .445 .175,00$ & 11,59 \\
\hline 1995 & $6.103 .134 .908,00$ & 11,38 \\
\hline 1996 & $7.806 .427 .374,00$ & 12,34 \\
\hline 1997 & $9.143 .412 .051,00$ & 13,21 \\
\hline 1998 & $9.322 .295 .898,00$ & 13,22 \\
\hline 1999 & $8.097 .858 .443,00$ & 10,73 \\
\hline 2000 & $9.394 .421 .653,00$ & 11,03 \\
\hline 2001 & $10.393 .177 .320,00$ & 10,68 \\
\hline 2002 & $11.336 .606 .377,00$ & 10,33 \\
\hline 2003 & $11.911 .317 .336,00$ & 9,11 \\
\hline
\end{tabular}

Fonte: 1) Balanço Geral do Estado do Rio Grande do Sul (1970-2003). Porto Alegre: Secretaria da Fazenda.

Notas: 1) DT é a despesa total que inclui o serviço da dívida (=amortização + pagamento de juros);

2) $\mathrm{gg}_{\mathrm{t}}=\mathrm{DT} / \mathrm{PIB}$; 3 ) a preços correntes. 